\title{
Serine and 1-carbon metabolism are required for HIF-mediated protection against retinopathy of prematurity
}

\author{
Charandeep Singh, ${ }^{1}$ George Hoppe, ${ }^{1}$ Vincent Tran, ${ }^{1}$ Leah McCollum, ${ }^{1}$ Youstina Bolok, ${ }^{1}$ Weilin Song, ${ }^{1}$ \\ Amit Sharma, ${ }^{1}$ Henri Brunengraber, ${ }^{2}$ and Jonathan E. Sears ${ }^{1,3}$ \\ 'Cole Eye Institute, Cleveland Clinic, Cleveland, Ohio, USA. ²Department of Nutrition, Case Western Reserve University, \\ Cleveland, Ohio, USA. ${ }^{3}$ Cardiovascular and Metabolic Sciences, Cleveland Clinic, Cleveland, Ohio, USA.
}

\begin{abstract}
We determined which metabolic pathways are activated by hypoxia-inducible factor 1-mediated (HIF-1-mediated) protection against oxygen-induced retinopathy (OIR) in newborn mice, the experimental correlate to retinopathy of prematurity, a leading cause of infant blindness. HIF-1 coordinates the change from oxidative to glycolytic metabolism and mediates flux through serine and 1-carbon metabolism (1CM) in hypoxic and cancer cells. We used untargeted metabolite profiling in vivo to demonstrate that hypoxia mimesis activates serine/1CM. Both $\left[{ }^{13} \mathrm{C}_{6}\right]$ glucose labeling of metabolites in ex vivo retinal explants as well as in vivo $\left[{ }^{13} C_{3}\right]$ serine labeling of metabolites followed in liver lysates strongly suggest that retinal serine is primarily derived from hepatic glycolytic carbon and not from retinal glycolytic carbon in newborn pups. In HIF-1 $\alpha^{210 x / 2 l o x}$ albumin-Cre-knockout mice, reduced or near- 0 levels of serine/glycine further demonstrate the hepatic origin of retinal serine. Furthermore, inhibition of 1CM by methotrexate blocked HIFmediated protection against OIR. This demonstrated that 1CM participates in protection induced by HIF-1 stabilization. The urea cycle also dominated pathway enrichment analyses of plasma samples. The dependence of retinal serine on hepatic HIF-1 and the upregulation of the urea cycle emphasize the importance of the liver to remote protection of the retina.
\end{abstract}

Conflict of interest: The authors have declared that no conflict of interest exists.

Copyright: (c) 2019, American Society for Clinical Investigation.

Submitted: April 19, 2019

Accepted: June 13, 2019

Published: July 25, 2019.

Reference information: /CI Insight. 2019;4(14):e129398. https://doi. org/10.1172/jici.insight.129398.

\section{Introduction}

Retinopathy of prematurity (ROP) is the most common form of infant blindness worldwide, accounting for 150,000 blind children annually (1). Survival after premature birth requires oxygen supplementation that is paradoxically associated with toxicity to premature developing tissues, such as the lung alveoli, nephrons of the kidney, cerebral cortex, and retinal capillaries (2). The direct relationship of higher oxygen saturation to disease severity has placed the oxygen-sensitive transcription factor hypoxia-inducible factor (HIF) as a central mediator of retinovascular growth and hence protection against ROP because eyes that have retinovascular growth attenuation develop severe ROP (3-5). We have definitively demonstrated the safety and efficacy of HIF stabilization in the prevention of oxygen-induced retinopathy (OIR). This was achieved via inhibition of HIF- $\alpha$ catabolism through blockade of HIF prolylhydroxylase (HIF PHD), resulting in a protected phenotype for both retina and lung simultaneously by systemic HIF PHD inhibition (6-8).

HIF-1 mediates adaptive metabolic responses of cells to hypoxia by increasing flux through glycolysis and decreasing entry of glycolytic carbon into the tricarboxylic acid (TCA) cycle (9). This occurs through upregulation of the PDK1 gene encoding pyruvate dehydrogenase kinase, which inactivates pyruvate dehydrogenase (10). Reduced flux through oxidative phosphorylation by HIF-1 decreases reactive oxygen species production (10). HIF also increases serine synthesis and metabolism by upregulating phosphoserine aminotransferase, phosphoglycerate dehydrogenase (PHGDH), and mitochondrial serine hydroxymethyltransferase 2 (SHMT2), contributing to increased mitochondrial NADPH production (11-14). HIF-1 stabilization induces serine synthesis from the intermediary metabolite 3-hydroxypyruvate, which is found in retinas from hyperoxic animals cycled through the OIR model (15). The conversion of 3-hydroxypyruvate to 3-phosphoglycerate, the precursor to serine, is initiated by glyoxylate reductase/hydroxypyruvate reductase, also induced by $\operatorname{HIF-1}(15,16)$. 
Hypoxia mimesis can be created pharmaceutically through inhibition of HIF PHD (17). These $\alpha$-ketoglutarate-dependent ( $\alpha$-KG-dependent) enzymes are part of the superfamily of dioxygenases. PHDs split molecular dioxygen to (a) hydroxylate regulatory proline residues on target proteins and (b) oxidize $\alpha-K G$ to succinate and $\mathrm{CO}_{2}(18,19)$. There are multiple targets of PHDs; perhaps the most notable of these are the HIF transcription factors (19-21). Inhibition of dioxygenases even in hyperoxia can be induced using small-molecule carbonyl glycines that are antagonistic, competitive $\alpha-K G$ analogs $(22,23)$. The regulation of dioxygenase activity by $\alpha-\mathrm{KG}$ and oxygen renders these enzymes responsive to both oxygen tension and metabolic intermediates (24).

Transcriptional and knockout studies of animals treated with PHD inhibition have determined a unique liver-eye and liver-heart axis that directs "remote" protection against OIR and cardiac ischemia, respectively $(25,26)$. Here we used untargeted metabolite profiling to demonstrate that (a) retinal serine/ glycine concentrations and (b) activation of retinal serine/1-carbon metabolism (1CM) are hepatic HIF-1 dependent. This supports a metabolic phenotype of mice protected by pharmacologic HIF stabilization against oxygen toxicity. These results demonstrate the importance of 1CM to HIF-1-mediated protection against OIR and further implicate the liver as a mediator of remote protection.

\section{Results}

To determine which metabolic pathways are globally increased by HIF PHD inhibition in the liver and retina, we treated newborn mouse pups exposed to hyperoxia with either sham intraperitoneal (i.p.) injection of phosphate-buffered saline (PBS) or Roxadustat (RXD), an HIF PHD inhibitor (27). We then analyzed the metabolites in the plasma and retina from pups using untargeted liquid chromatography tandem-mass spectrometry (LC-MS/MS) analysis. Figure 1, A-D, shows the principal component analysis (PCA) of positive and negative modes from plasma and retina in hyperoxia and hyperoxia plus HIF stabilization. Untargeted metabolite profiling was used for an unbiased measurement of the polar metabolites present in the plasma and retina samples. Every sample was measured 2 times, once in positive mode and then in negative mode, using the ZIC-HILIC-ddMS2 method (zwitterionic-hydrophilic interaction liquid chromatography-data-dependent mass spectrometry). Data were analyzed using XCMS Online tool (28), and unsupervised PCA clearly indicated differences in metabolic features present in the plasma or retina harvested from controls and RXD-treated mice (Figure 1, A-D).

XCMS cloud plot analysis of plasma samples revealed changes in 613 (Supplemental Figure 1A; supplemental material available online with this article; https://doi.org/10.1172/jci.insight.129398DS1) and 398 (Supplemental Figure 1B) metabolic features with $P$ value less than or equal to 0.01 and fold change at least 1.5 in the positive and negative modes, respectively, when comparing hyperoxic control and RXD-treated hyperoxic mouse plasma samples. In contrast, retina cloud plot analysis showed 82 (Supplemental Figure 1C) and 23 (Supplemental Figure 1D) metabolic features with $P$ value less than or equal to 0.01 and fold change at least 1.5 , in positive and negative modes, respectively.

To define the most important biochemical pathways that produced these metabolic differences, we performed system-level analysis using XCMS Online systems biology tool. Systems biology analyses of the metabolomics data in XCMS Online are based on mummichog analysis of the metabolite features (29). The conventional approach to metabolomics analysis is to first identify metabolites based on the exact mass of the compound and then to map them onto a metabolic network of the organism under investigation. However, mummichog analysis is a recursive approach where metabolite feature identification and mapping onto metabolic network are considered correlated events. All the features differing between 2 conditions are mapped onto multiple pathways specific to an organism of interest (in our case the mouse), and the pathways with the best fit are displayed as outputs (Figure 2, A-D, and Supplemental Tables 1-4). Of those, branches of serine/1CM and urea cycle populated the pathway enrichment graphs. For example, pathways such as glycine-betaine, glycine, glutathione, creatine, folate, serine, and purine all need serine as a precursor. Similarly, citrulline, arginine, nitric oxide, and glutamine all are interlinked by the urea cycle. This analysis definitively demonstrated induction of serine/1CM and urea pathways by RXD.

We next analyzed data manually and looked at metabolites within the serine and urea pathways (see Supplemental Figures 2 and 3 and Figure 2E, selected metabolites). MS1 peak areas of the compounds confirmed by MS2 library matches and manual analysis were plotted for all 3 conditions, i.e., normoxia, hyperoxia, and hyperoxia plus RXD (Figure $2 \mathrm{E}$ ). We found $50 \%$ or higher increased levels of plasma serine, glycine, hypotaurine, cystathionine, methionine, LysoPC18:2, and taurine from RXD-treated animals 

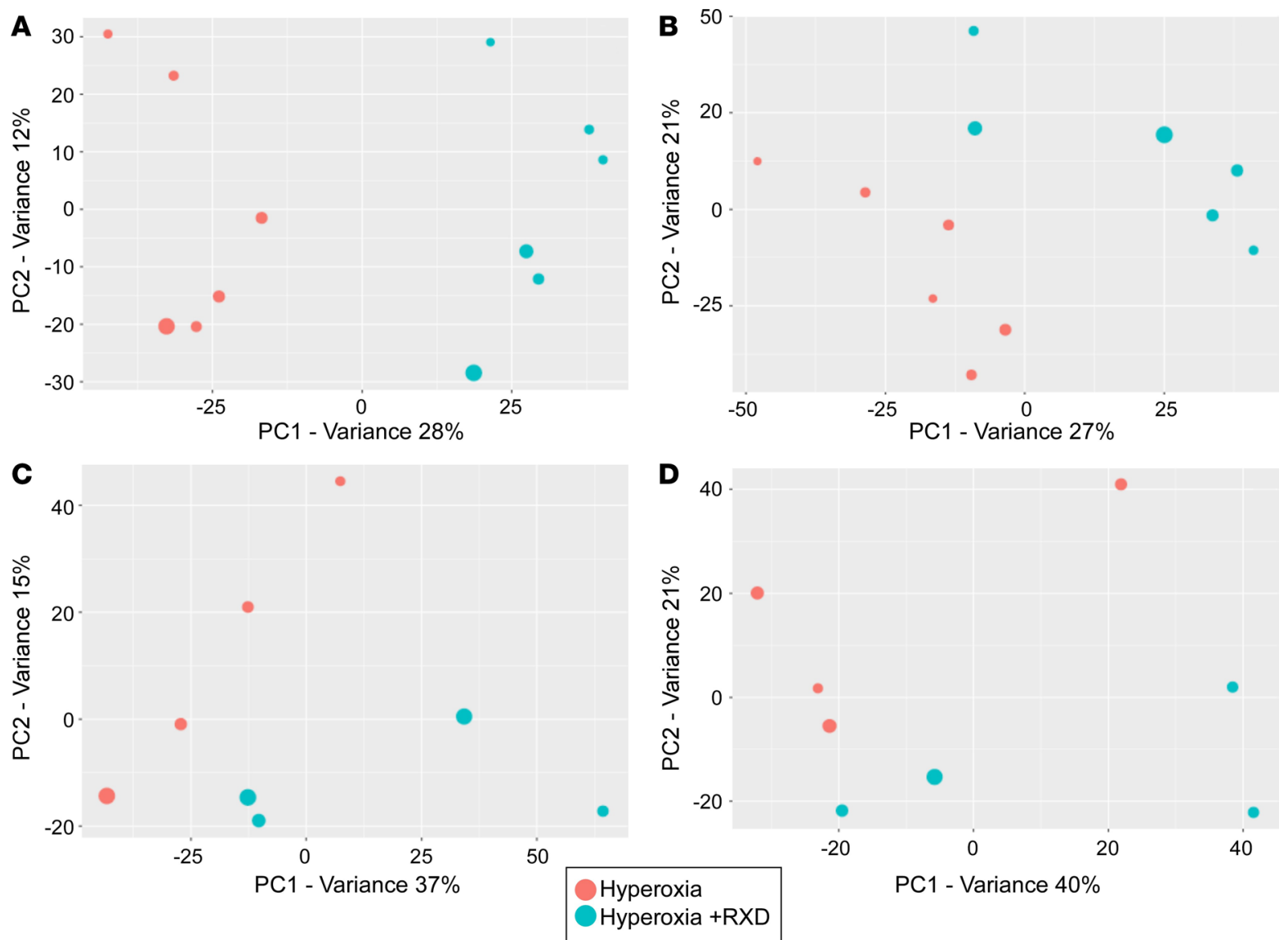

Figure 1. Multivariate statistical analysis of metabolite features indicates difference in PBS (control) versus Roxadustat (treated) hyperoxic mice, in plasma and retina metabolome. All the data in this figure are from mice dissected on postnatal day 10. Metabolites from retina ( $n=4$, each group) and plasma ( $n=6$, each group) of these mice were extracted and analyzed using LC-MS/MS. Multidimensional data were analyzed using PCA (A-D). The size of the dot represents DModX value for each sample. DModX represents distance of each observation to the model plane and helps in determination of potential outliers, which in our case were absent. (A) PCA score plot of positive ionization mode plasma metabolite features of principal component 1 (PC1) versus PC2. (B) PCA score plot of negative ionization mode plasma data. (C) PCA score plot of positive ionization mode retina data. (D) PCA score plot of negative ionization mode retina data. RXD, Roxadustat.

in hyperoxia compared with control hyperoxic animals (Figure 2E, selected metabolites; Supplemental Figure 2, plasma; and Supplemental Figure 3, retina). In addition, metabolites of the urea cycle were increased under HIF stabilization. For example, $N$-acetylglutamate increased 4-fold in plasma, while orotate, citrulline, arginine, aspartate, and glutamine were at least 50\% increased in the RXD-treated mouse plasma, as demonstrated in Supplemental Figure 2. $\mathrm{N}$-acetylglutamate is an allosteric activator of carbamoyl phosphate synthetase 1 , the first enzyme in the urea cycle pathway.

$\left[{ }^{13} \mathrm{C}_{3}\right]$ serine atom transitions in $1 \mathrm{CM}$ are summarized in Figure $3 \mathrm{~A}$. To test the relevance of $1 \mathrm{CM}$ to protection against OIR, we treated mouse pups with methotrexate (MTX) to inhibit dihydrofolate reductase, in the first (hyperoxic) phase of OIR from postnatal day 6 (P6) to P11, to prevent use of carbon extracted from serine. MTX-treated animals had a decreased protective response to HIF stabilization when comparing control-injected PBS (Figure 3D) to RXD (Figure 3E) or RXD plus MTX (Figure 3F). In animals in which serine use was inhibited by MTX, there was a $55 \%$ reduction in protection manifested by an increase in vaso-obliteration, as quantified in Figure 3B. MTX had no effect on pathological angiogenesis or neovascularization (Figure 3F, quantified in Figure 3C), presumably because it was administered only in phase 1 (hyperoxia). In addition, MTX did not increase vaso-obliteration in PBS controls, demonstrating that it blocked protection by RXD but was not additive to hyperoxia likely because hyperoxia had already downregulated $1 \mathrm{CM}$. The $\mathrm{LD}_{50}$ established for MTX is around $94 \mathrm{mg} / \mathrm{kg}$ of mice weight (30), which is around 470 times higher than the dose used in our experiment. To test whether MTX inhibits serine to glycine conversion at this low dose we used in our experiments, we injected $\left[{ }^{13} \mathrm{C}_{3}\right]$ serine into the 
A

3

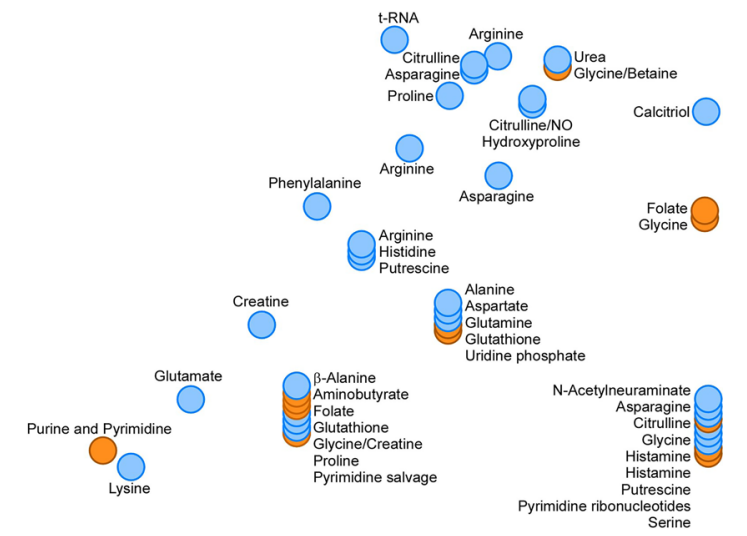

B

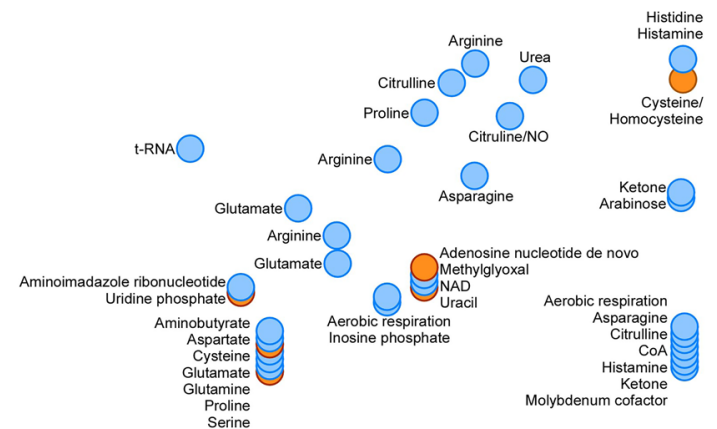

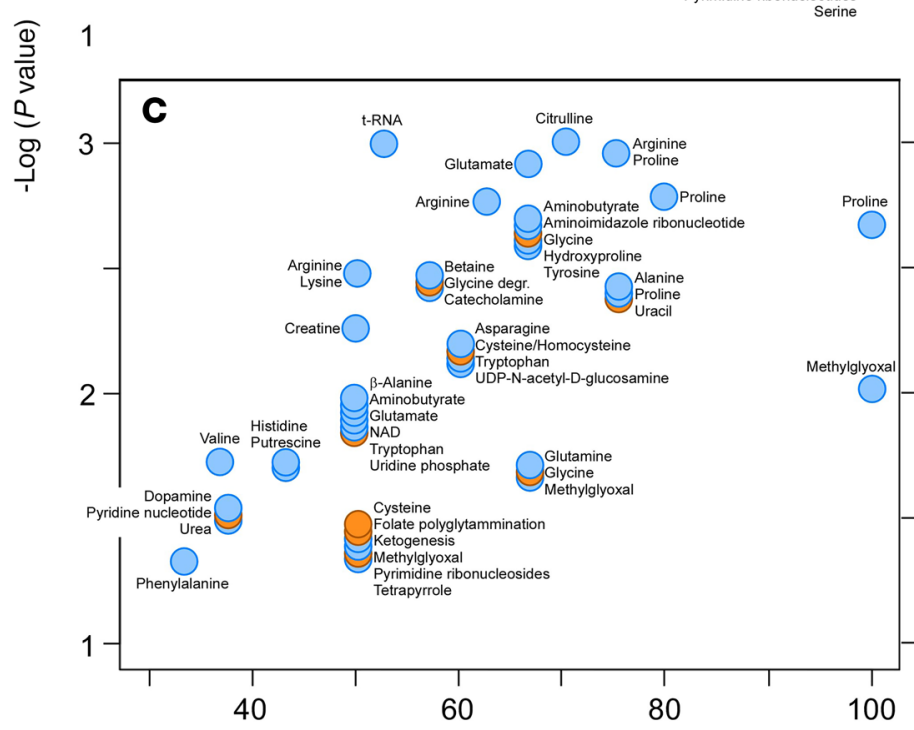

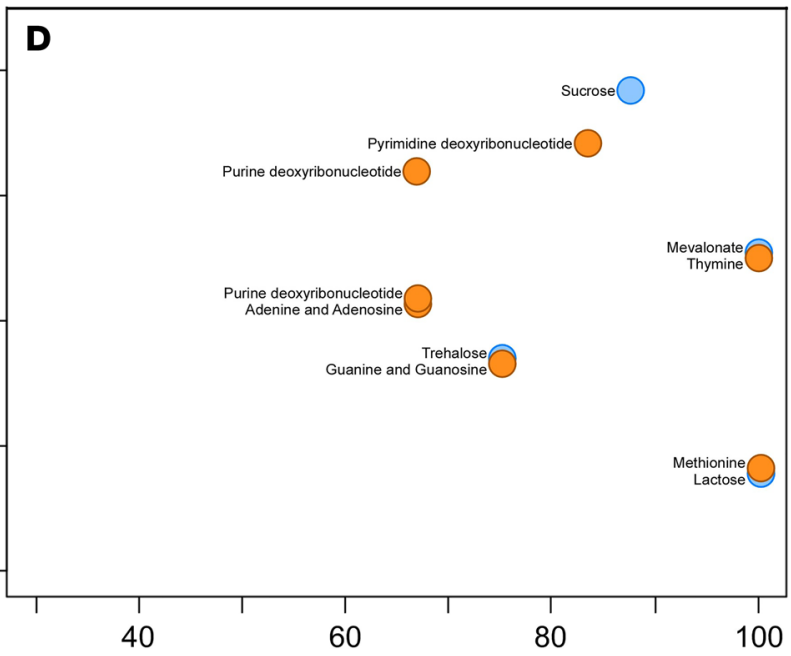

Average Overlap (metabolites) Percentage

E

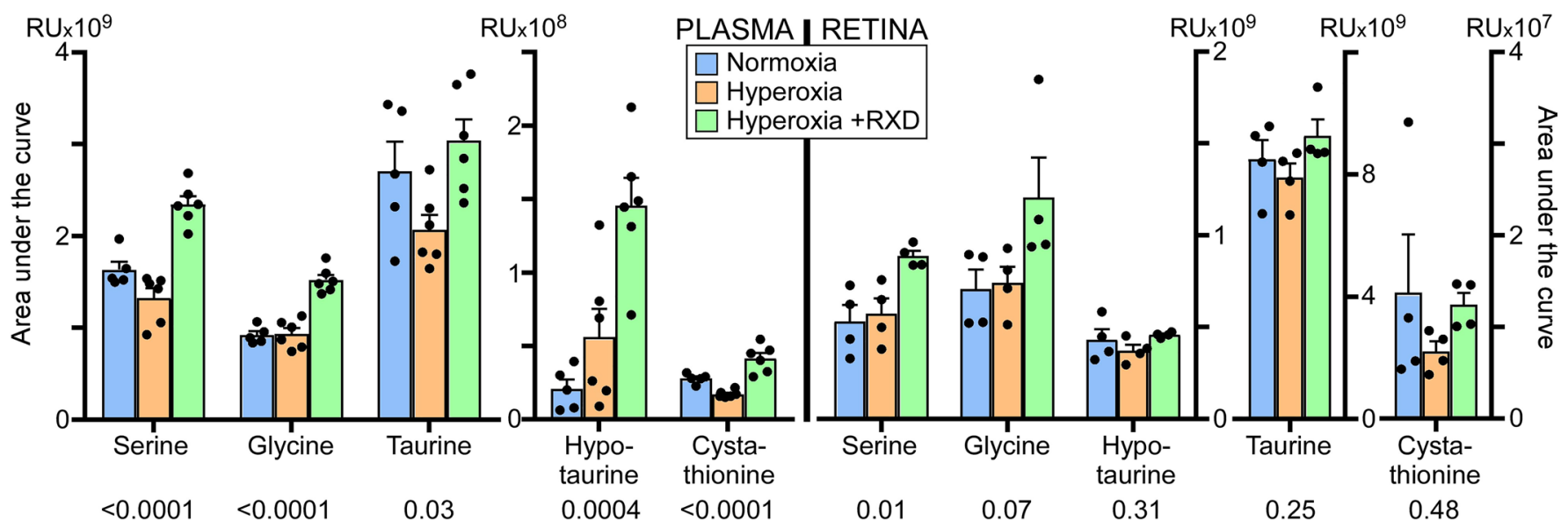

One-way ANOVA

Figure 2. Pathway cloud plot depicting various metabolic pathways affected by RXD. All the data in this figure are from mice dissected on postnatal day $10(\mathbf{A}-\mathbf{D})$, and bar graph of annotated metabolites (E) shows that PHi targets the serine/1C pathway. Metabolite features were analyzed through a pathway prediction algorithm in XCMS Online tool, and statistically significant pathways with $P$ value less than 0.05 are depicted in these plots. Data were projected onto a mouse-specific database available on XCMS. Please also see pathway tables in Supplemental Tables 1-4. (A) Positive mode plasma data. (B) Negative mode plasma data. (C) Positive mode retina data. (D) Negative mode retina data. Serine /1CM metabolic pathways are highlighted in orange. Targeted data analysis was performed on a few metabolites from the serine/1C pathway. Identities of metabolites were confirmed using MS1 exact mass and MS2 fragmentation pattern. (E) Selected plasma metabolites ( $n=6$ for each condition) and retina metabolites ( $n=4$ for each condition). $P$ values are shown below the $x$ axis. 

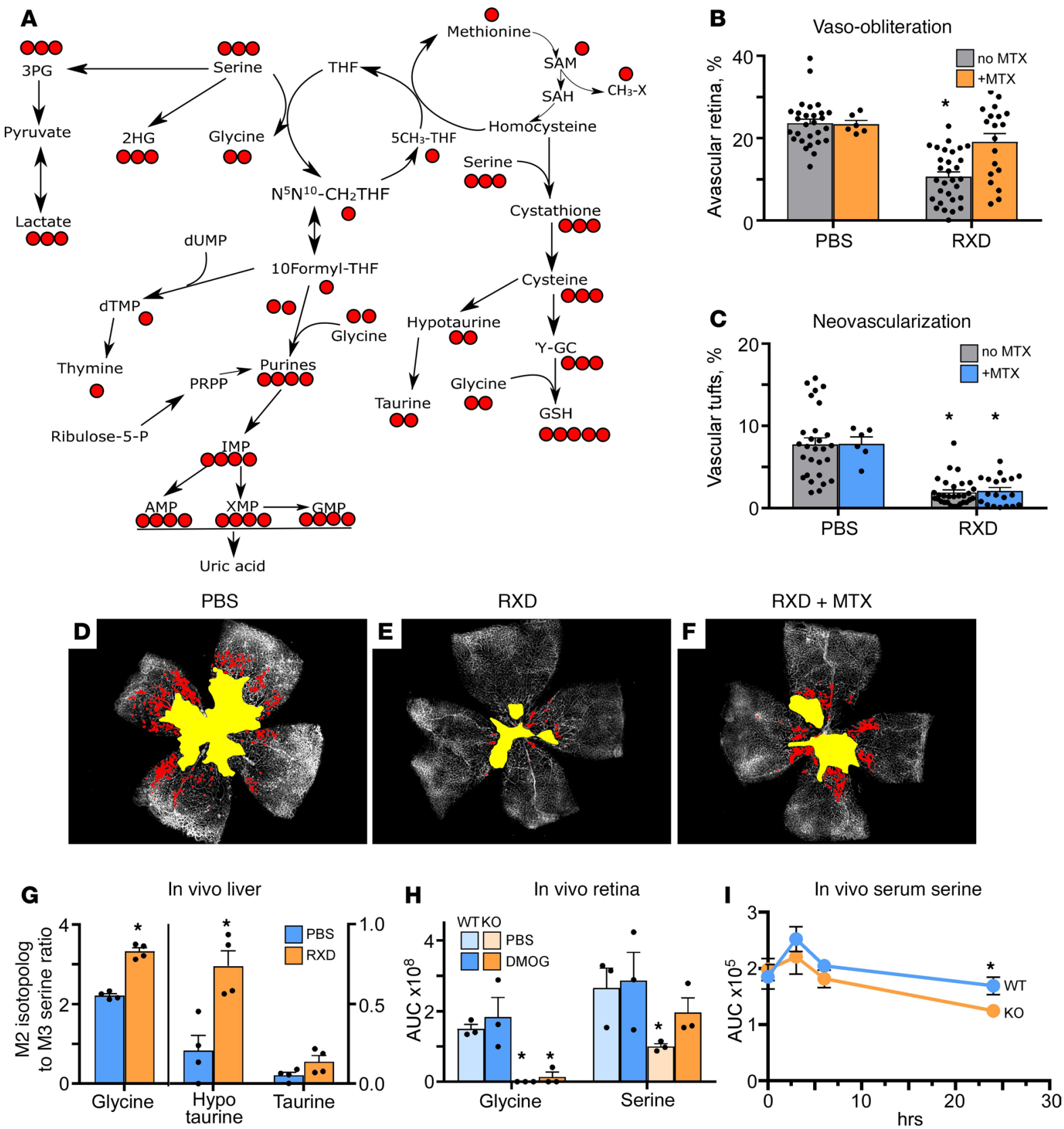

Figure 3. Hepatic 1CM drives protection against OIR. (A) Schematic showing atom transitions in all aspects of 1CM. (B) Quantification of newborn mice treated with PBS or PBS plus methotrexate (MTX) compared with Roxadustat (RXD) or RXD plus MTX. There was increased vaso-obliteration in the RXD plus MTX group. MTX did not worsen the control group but did worsen the RXD group, showing that hyperoxia downregulates 1CM to such an extent that it is not additive to creating pathology. Under HIF stabilization, 1CM is upregulated and this is blocked by MTX. (C) Quantification of pathological angiogenesis or neovascularization shows that 1CM has no effect on proliferation presumably because it was administered only in phase 1. (D) Representative flat mount from control PBSinjected newborn mouse shows capillary dropout (yellow) and neovascularization (red). (E) Mouse pup retinal flat mount protected by HIF stabilization using RXD. (F) MTX plus RXD (RXD + MTX) demonstrates reduction in protection when $1 C M$ is blocked by dihydrofolate reductase. (C) $\left[{ }^{13} C_{3}\right]$ serine injected subcutaneously was followed in liver extracts, demonstrating conversion to M2 glycine, M2 hypotaurine, and M2 taurine, all metabolites of 1CM. (H) The change in retinal serine and glycine using a hepatic-specific HIF stabilizer in wild-type (WT) versus HIF-1 $\alpha^{210 x / 210 x}$ albumin-Cre-knockout (HIF-1 $\left.\alpha-K 0\right)$ mice. ( $n=6$ animals in each group.) (I) Serum serine and glycine levels in the WT versus HIF-1 $\alpha^{210 x / 210 x}$, DMOG-injected mouse metabolite extracts. Metabolites were extracted from the serum at different time points after DMOC i.p. injections. ${ }^{*}$, 2-tailed $t$ test $P$ values less than 0.05 .

pups and measured serine to glycine conversion. Serine to glycine conversion was indeed decreased in liver and retina in the MTX-treated mice pups (Supplemental Figure 4, A-L).

To test whether the liver increased serine metabolism under HIF stabilization, we performed in vivo dynamic labeling experiments on hyperoxic PBS- and RXD-treated mouse pups by injecting $\left[{ }^{13} \mathrm{C}_{3}\right]$ 
serine subcutaneously and measured downstream metabolites of $1 \mathrm{CM}$ in liver (Figure 3G). Liver extracts demonstrate that RXD increased hepatic serine use, demonstrated by increased ratios of M2 glycine/M3 serine, M2 taurine/M3 serine, and M2 hypotaurine/M3 serine (Figure 3G) 1 hour after subcutaneous injection of serine tracer.

We next determined whether metabolites of retinal 1CM are dependent on hepatic HIF-1 stabilization by measuring retinal serine/glycine concentrations in the hepatic HIF-1-knockout mouse (conditional Crelox HIF-1 $\alpha$ [HIF-1 $\alpha^{210 x / 2 l o x}$ albumin-Cre] hepatic knockout) (HIF-1-KO, see Supplemental Figure 5 for tissue specificity) with and without HIF stabilization. To ensure that we were analyzing only the hepatic contribution to increases in retinal serine, we used dimethyloxalylglycine (DMOG), a widely studied $\alpha-\mathrm{KG}$ antagonist and nonselective inhibitor of HIF PHD. We have definitively demonstrated that DMOG can protect only the wild-type mouse but not the hepatic HIF-1-KO, demonstrating the HIF-1 dependence of the liver to eye protection using DMOG (25). The liver selectivity of DMOG is likely a consequence of DMOG's instability and inability to survive intact after a first pass through the liver. In cell culture, DMOG is rapidly hydrolyzed to either $n$-oxalylglycine or methyl oxalylglycine (31). To prove that DMOG does not enter plasma, we used LC-MS/MS to analyze plasma from animals treated with either DMOG or RXD injected i.p. Supplemental Figure 6A, top, shows the DMOG standard compared to middle and lower in Supplemental Figure 6A, which demonstrates that DMOG does not survive the first pass through the liver because there was no peak with exact mass and retention time corresponding to that of the authentic DMOG standard. In contrast, Supplemental Figure 6B shows RXD standard (top) and peak in plasma and retina with same $\mathrm{m} / z$, retention time, and MS2 spectrum in the plasma (middle) and retina (lower) of the mice injected with RXD. Supplemental Figure 6C shows preservation of RXD in plasma and retina measured as area under the curve, further confirmed by MS2 analysis (Supplemental Figure 6) in plasma and retina, which reveals identical fragmentation patterns. DMOG, unlike RXD, targets only the liver and cannot reach the retina. These data support the observation that systemic HIF-1 stabilization by DMOG protects only the wild-type mouse from oxygen toxicity but does not protect the hepatic HIF-1-KO (25). We therefore next used the hepatic HIF-1KO mice treated with DMOG to test whether metabolites of 1CM were reduced in the hepatic HIF-1-KO. Measurements of retinal serine and glycine in the hepatic HIF-1-KO mice (Figure $3 \mathrm{H}$ ) showed that hepatic HIF-1 modulated retinal serine and glycine concentrations. Both retinal serine and glycine were decreased in the HIF-1-KO compared with WT mice in the same hyperoxic conditions with HIF stabilization by DMOG. We also measured serine levels in serum samples from DMOG-injected WT and HIF-1-KO time course. Serine levels were same at the start of the time course, 0 minutes after DMOG injection. The WT demonstrates higher levels of serum serine than the $\mathrm{KO}$ at 24 hours after injection with DMOG, with a $t$-test $P$ value of 0.01 (Figure 3I).

Our hepatic HIF-1-KO mouse studies clearly demonstrate that retinal serine/glycine depends on liver HIF-1. We next measured the glycolytic fluxes in retina by stable isotope-based metabolomics. The atom transitions of $\left[{ }^{13} \mathrm{C}_{6}\right]$ glucose are provided in Figure 4A. HIF stabilization induced a very small (less than $10 \%$ ) increase in the glycolytic fluxes in retinal explants, measured as M3 isotopolog of lactate, in RXD-treated versus untreated retinal explants (Figure 4, B and C). There was a very high baseline rate of glucose to lactate conversion by retina found to be $332 \mathrm{nmol} / \mathrm{h} / \mathrm{mg}$ (wet-weight basis). This value is in the range of that previously reported $(32,33)$. Lactate measurements were necessary to confirm that glucose was oxidized in these experiments. In contrast, there was no enrichment of either M3 serine or M2 glycine (Figure 4, D and E), indicating that retina does not use glycolytic carbon to synthesize serine; if so, serine would be labeled M3 and derived from M3 3-phosphoglycerate. Lactate M3 labeling is clear proof of M3 labeling of 3-phosphoglycerate, yet there was no shuttling of 3-phosphoglycerate to the serine pathway in retinal explants with or without HIF stabilization. The incubation also included nitrogenous substrate (glutamine) that would have allowed synthesis of labeled amino acids from glucose. The small amount of M1 serine may imply anaplerosis from M3 pyruvate and production of retinal serine by decarboxylation of malate, although this putative process would have likely yielded other isotopologs (Figure 4D).

\section{Discussion}

Our data demonstrate hepatic HIF-1-dependent regulation of the urea cycle and serine/1CM in a proangiogenic strategy to protect retinal blood vessels. These findings suggest a metabolic mechanism to explain how remote protection induced by hepatic HIF PHD inhibition could protect peripheral capillary beds, inasmuch as both pathways rely on hepatic metabolism. Our approach of using analysis of metabolic 

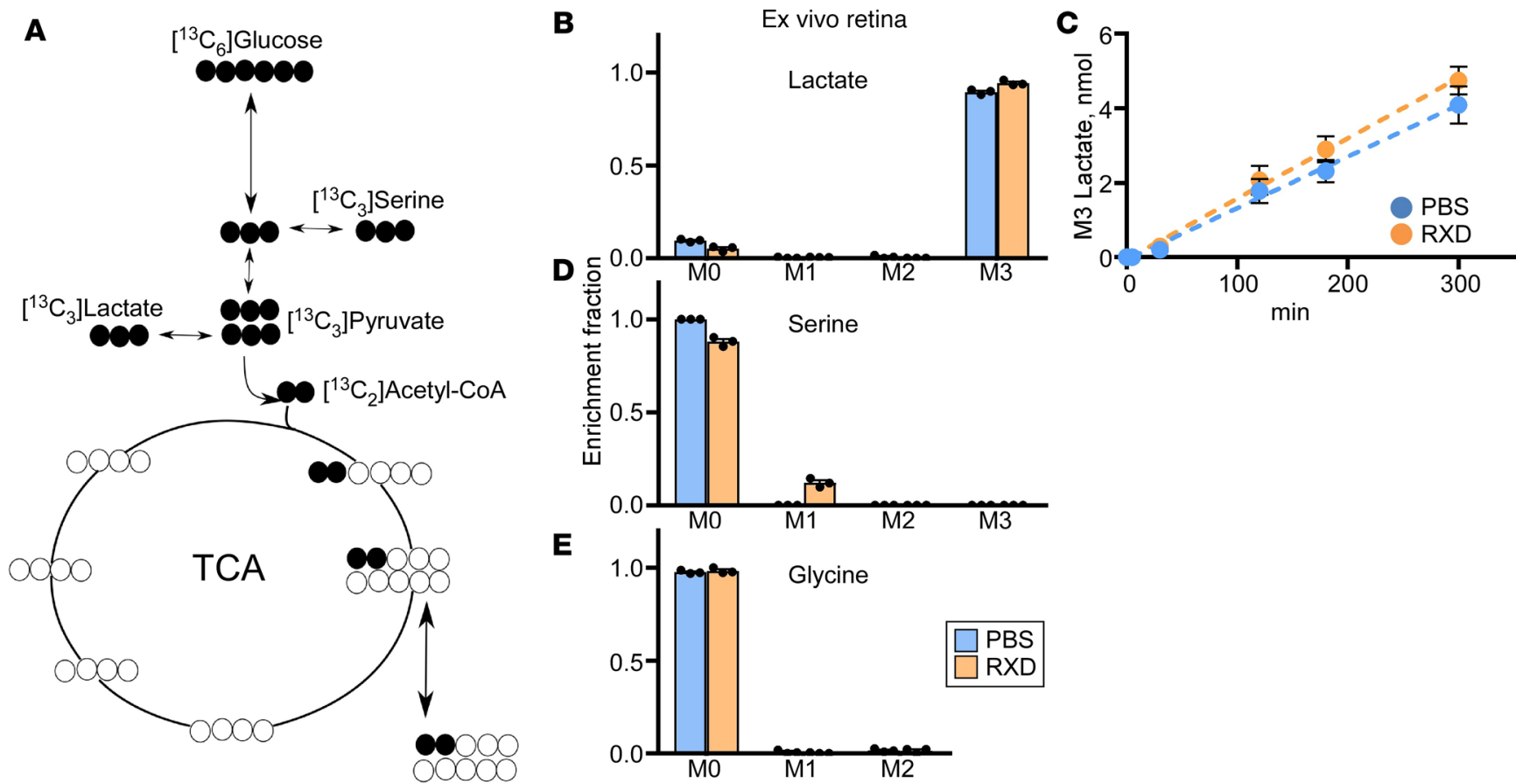

Figure 4. Ex vivo $\left[{ }^{13} C_{6}\right]$ glucose labeling of retina and in vivo $\left[{ }^{13} C_{3}\right]$ serine labeling of liver demonstrates that retinal serine is derived from liver. Retinal explants were incubated in Krebs-Ringer buffer $(\mathrm{KRB})$ medium containing $5 \mathrm{mM}\left[{ }^{13} \mathrm{C}_{6}\right]$ glucose and $1 \mathrm{mM}\left[{ }^{12} \mathrm{C}\right]$ glutamine. As expected, a very high rate of $\left[{ }^{13} \mathrm{C}_{6}\right]$ glucose to $\left[{ }^{13} C_{3}\right]$ lactate was observed, whereas no comparable ${ }^{13} \mathrm{C}$ carbon enrichment was seen in serine or glycine, indicating that glycolytic flux in retina is not responsible for serine or glycine formation. (A) Schematic showing expected labeling from the first-round carbon flow from glucose to glycolytic and TCA cycle intermediates when labeled with $\left[{ }^{13} \mathrm{C}_{6}\right]$ glucose. (B) Media labeling of the lactate by retina sampled 5 hours after incubation with [ $\left.{ }^{13} \mathrm{C}_{6}\right]$ glucose. (C) Lactate production rate was not significantly changed between PBS controls and RXD-treated retina explants. (D) Intracellular labeling of serine in retina shows that retina did not synthesize serine from glucose because $\mathrm{MO}$ was the dominant metabolite sampled 5 hours after incubation with $\left[{ }^{3} \mathrm{C}_{6}\right]$ glucose. (E) Intracellular labeling of glycine, like serine, was $\mathrm{MO}$ and hence not derived from glucose sampled 5 hours after incubation with $\left[{ }^{13} \mathrm{C}_{6}\right]$ glucose.

features derived from in vivo models with and without small molecules that stabilize HIF demonstrates a different perspective of Systems Pharmacology, which ordinarily relies on transcriptional analysis. Our previous study of this topic does reveal specific upregulation of SHMT2, methylene tetrahydrofolate dehydrogenase 2, and MTHDF1L (MTHDF-like), indicating the HIF-1 induction of mitochondrial 1CM that produces glycine and ultimately formate for regeneration of serine in the cytosol (Gene Expression Omnibus GSE74170; ref. 16).

Although our experiments clearly demonstrate that whole retinal explants do not synthesize serine from glycolytic carbon, endothelial cells in culture, such as primary hepatic endothelial cells and human umbilical vein endothelial cells, produce serine (34). Tamoxifen-induced KO of PHGDH in endothelial cells systemically also induces decreased retinovascular growth (34). Retinal endothelial cells also synthesize serine if they are both HIF stabilized and given 3-hydroxypyruvate simultaneously (15). However, our in vivo experiments strongly suggest that the liver is primarily responsible for serine/glycine synthesis, at least in the newborn mouse. This correlates to experiments in sheep fetuses, where the flux of serine is measured at $2700 \mu \mathrm{mol} / \mathrm{h} / \mathrm{kg}$. The sheep fetal liver is primarily responsible for serine production from glycine taken up from the placenta (35).

Loss of function of PHGDH, a rate-limiting enzyme for serine synthesis, is associated with macular telangiectasia (MacTel; ref. 36). Yet, the average age of onset of MacTel is 50 to 60 years (37), suggesting that if $1 \mathrm{CM}$ is relevant to disease pathogenesis, it is associated with senescence or perhaps compensated by systemic sources of serine/glycine. Our hepatic HIF-1-KO model was not completely devoid of retinal serine, but retinal glycine levels were near 0 .

A natural question is whether serine supplementation can protect against OIR. We have tried high doses of i.p. serine and found no effect (data not shown). We considered that serine was catabolized too rapidly to confer protection or that protection requires upregulation of a specific transporter, such as mitochondrial sideroflexin 1, a multipass mitochondrial membrane protein (38). In addition, 1CM may not respond solely to substrate concentrations but require upregulation of HIF-1-dependent enzymes within the cycle. 
HIF-1 stabilization at the beginning of the OIR cycle induced ureagenesis. In contrast, without HIF stabilization, ureagenesis is upregulated at the end of the OIR cycle (39). Pathological angiogenesis, termed neovascularization, occurs by way of a 2-step hypothesis (40-42). Phase 1 is the initiating event created by hyperoxia that leads to vascular obliteration and retinovascular growth attenuation. Neovascularization, associated with phase 2 at the end of the OIR cycle, is a reaction to oxygen-induced ischemia that creates severely hypoxic retina as mice are returned to room air after hyperoxia, or in the case of infants, as oxygen supplementation is withdrawn as these severely premature infants survive. It is interesting that an increase in the urea cycle pathway, normally seen in the whole-eye metabolome of phase 2 OIR and in the vitreous cavity of patients with diabetes (39), was shifted to phase 1 by HIF stabilization. If this represents part of vasoproliferation or vascular repair, it is beneficial to shift this response to phase 1 using hypoxia mimesis (HIF stabilization) because this is exactly when a proangiogenic response would be protective.

In conclusion, untargeted metabolite profiling in combination with pharmaceutical hypoxic preconditioning in vivo demonstrates that (a) systemic HIF stabilization requires $1 \mathrm{CM}$ to protect against OIR and (b) retinal serine/glycine concentrations are hepatic HIF-1 dependent.

\section{Methods}

Reagents. LC-MS-grade methanol (HiPerSolv Chromanorm; BDH VWR International) and chloroform (LiChrosolv; MilliporeSigma) were used for all the metabolite extractions. Stable isotopes were purchased from Cambridge Isotope Laboratories and were at least 99\% pure. MTX was purchased from Cayman Chemicals. $N$-methyl- $N$-(trimethylsilyl)trifluoroacetamide (MSTFA) was purchased from Macherey-Nagel and MSTFA with 1\% trimethylchlorosilane (TMCS) was purchased from Thermo Fisher Scientific. All the chemicals and metabolite standards were purchased from MilliporeSigma. KRB medium was prepared fresh as per recipe provided in Du, Linton, and Hurley (43). Hanks' balanced salt solution (HBSS) and PBS were purchased from Cleveland Clinic Media Lab.

Ex vivo retinal explant and in vivo whole-mouse isotopic labeling. For isotopic labeling experiments, retinas were dissected out while keeping eyes in ice-cold KRB medium containing $15 \mathrm{mM}$ glucose. Samples were then washed with PBS and centrifuged at $300 \mathrm{~g}$ at room temperature for 2 minutes to remove the PBS. The retina samples were then carefully transferred to $\mathrm{KRB}$ medium containing $5 \mathrm{mM}^{13} \mathrm{C}_{6}$ glucose, $1 \mathrm{mM}{ }^{12} \mathrm{C}$ glutamine, and 10\% dialyzed FBS. Media samples were obtained at 0, 5, 30, 120, 180, and 300 minutes; retinal explants were harvested at 300 minutes and metabolites extracted as described below. To perform the in vivo labeling of whole mice, pups were separated on P10 of the OIR model from their mother 6 hours after RXD injection and then injected with $80 \mu \mathrm{L}$ of $10 \mathrm{mg} / \mathrm{mL}$ subcutaneous injections of $\left[{ }^{13} \mathrm{C}_{3}\right]$ serine; mouse tissues were taken out at 1 hour after serine injections and metabolites extracted as described below.

MTX isotopic labeling experiment. C57BL/6J WT mice were kept in hyperoxia from P10 to P12. RXD was administered on P10 and P12, and MTX $0.2 \mu \mathrm{g} / \mathrm{mg}$ of body weight was injected every day. On P12, mice were injected the last dose of MTX and separated from nursing dam for 1 hour. Thirty minutes after MTX injections, $80 \mu \mathrm{L}$ of $6 \mathrm{mg} / \mathrm{mL}\left[{ }^{13} \mathrm{C}_{3}\right]$ serine was injected subcutaneously, and mice were placed back in the hyperoxic chamber. At the end of the experiment, mice were anesthetized with ketamine/xylazine mix and euthanized to dissect livers. Twenty milligrams of liver was used to extract metabolites. Samples were processed as described later in the text, and $300 \mu \mathrm{L}$ of upper phase from metabolite extraction was dried per sample. Metabolites were measured with a gas chromatography-mass spectrometry (GC-MS) scan method very similar to as described later in the next section and were measured on ZORBAX DB-5ms $+10 \mathrm{~m}$ DuraGuard Capillary Column (Agilent) and with split 1:10 injection.

Collection of tissues from mice and metabolite extraction. To extract the blood samples, mice were anesthetized with ketamine/xylazine mix, and then blood was extracted from the heart of the animal with a 27.5-gauge needle fixed onto a 1-cc heparinized syringe. The extract was added to $1.5-\mathrm{mL}$ tubes containing $1.5 \mu \mathrm{L}$ of heparin 10,000 USP units/mL (Hospira) and stored on ice until further use. Eyes were enucleated and retinas were dissected in cold HBSS under the microscope. Tubes containing retina were immediately frozen in liquid nitrogen. Liver was also quickly dissected and snap-frozen in liquid nitrogen as well. Plasma was obtained from blood samples by centrifuging samples at $1100 \mathrm{~g}$ at $4^{\circ} \mathrm{C}$ for 20 minutes.

Metabolites from retina ( 2 retinas were combined per tube) were extracted using methanol/chloroform/water phase separation. Briefly, $400 \mu \mathrm{L}$ of $-20^{\circ} \mathrm{C}$ cold methanol was added to $400 \mu \mathrm{L}$ of $4^{\circ} \mathrm{C}$ water. Samples were then briefly sonicated and $400 \mu \mathrm{L}$ of $-20^{\circ} \mathrm{C}$ cold chloroform was added. Samples were then vortexed on a thermomixer (Thermomixer C; Eppendorf) at $4^{\circ} \mathrm{C}$ for 20 minutes at $1400 \mathrm{rpm}$ and were then 
centrifuged at $15,000 \mathrm{~g}$ at $4^{\circ} \mathrm{C}$ for 5 minutes, following which the upper phase containing polar metabolites was separated into fresh vials and injected directly into the LC-MS or dried for the GC-MS analysis.

Metabolites from plasma were extracted by adding $20 \mu \mathrm{L}$ of plasma to $500 \mu \mathrm{L}$ of $50 \%$ cold acetonitrile. Samples were centrifuged at $15,000 \mathrm{~g}$ for 10 minutes, and $400 \mu \mathrm{L}$ supernatant was carefully removed into fresh tubes without disturbing the cell pellet. Supernatants were either injected directly into the LC-MS or $300 \mu \mathrm{L}$ dried for the GC-MS analysis.

Metabolites from serum were extracted by adding $10 \mu \mathrm{L}$ of serum using the same protocol as described above for plasma samples. Three hundred microliters of supernatant was dried for the GC-MS analysis. Metabolites were measured with a GC-MS scan method very similar to as described later in the next section and were measured on ZORBAX DB-5ms $+10 \mathrm{~m}$ DuraGuard Capillary Column and with split 1:10 injection.

Metabolites from media in which retinal explants were cultured were extracted by diluting $15 \mu \mathrm{L}$ of media with $15 \mu \mathrm{L}$ of $-20^{\circ} \mathrm{C}$ cold methanol. Media samples were centrifuged at $15,000 \mathrm{~g}$ at $4^{\circ} \mathrm{C}$ for 5 min utes, following which $20 \mu \mathrm{L}$ of sample was dried for derivatization and GC-MS measurement.

Metabolites from liver were extracted using methanol/chloroform/water protocol as described above but with slight differences. Briefly, $20 \mathrm{mg}$ of liver sample was weighted per tube, crushed with disposable pestle, and added to $50 \mu \mathrm{L}$ of $50 \%$ methanol $\left(-20^{\circ} \mathrm{C}\right.$ cold $)$. Samples were sonicated on ice to break open the cells, and additional methanol, water, and chloroform were added to get to a final mixture with $400 \mu \mathrm{L}$ of each solvent. Samples were then vortexed on a thermomixer at $1400 \mathrm{rpm}$ at $4^{\circ} \mathrm{C}$ for 20 minutes, followed by centrifugation at $15,000 \mathrm{~g}$ at $4^{\circ} \mathrm{C}$ for 5 minutes. Supernatant was removed into a fresh $1.5-\mathrm{mL}$ tube and diluted $1: 1$ with $50 \%$ of $-20^{\circ} \mathrm{C}$ methanol. The diluted sample, $360 \mu \mathrm{L}$ volume, was dried and derivatized for GC-MS measurement.

For GC-MS measurements, samples were dried under vacuum at $-4^{\circ} \mathrm{C}$ with subsequent drying at $30^{\circ} \mathrm{C}$ for 10 minutes to ensure no trace of moisture was present in the samples. Samples were then derivatized using a 2-step protocol. First, the sample was derivatized with $25 \mu \mathrm{L}$ of $40 \mathrm{mg} / \mathrm{mL}$ of $\mathrm{MTX}$ in pyridine at $45^{\circ} \mathrm{C}$ for 30 minutes. We followed up with an additional derivatization step by adding $75 \mu \mathrm{L}$ of MSTFA (MSTFA $+1 \%$ TMCS was used in the liver stable isotope tracer experiment) at $45^{\circ} \mathrm{C}$ for 30 minutes. Samples were packed in airtight GC-MS vials, and $1 \mu \mathrm{L}$ of the sample was injected into the mass spectrometer. GC-MS was used in either electron ionization (EI) or chemical ionization (CI) with methane as a reagent gas.

LC-MS/MS measurement of polar metabolites. Untargeted metabolomics was performed on the UPLC (Vanquish, Thermo Fisher Scientific) connected to Q-exactive HF mass spectrometer (Thermo Fisher Scientific). Chromatographic separation was performed on SeQuant ZIC-HILIC column with dimensions $150 \times 2.1 \mathrm{~mm}$, $3.5 \mu \mathrm{m}, 100 \AA$ (Merck), attached to a precolumn SeQuant ZIC-HILIC with dimensions $20 \times 2.1 \mathrm{~mm}, 3.5 \mu \mathrm{m}$, $100 \AA$ (Merck). The LC method used for separation of metabolites using the ZIC-HILIC column was adapted from Singh et al. (44). Briefly, gradient of solvent A ( $0.1 \%$ formic acid in water) and solvent B $(0.08 \%$ formic acid in acetonitrile) was ramped from $80 \%$ B to $35 \%$ B in 23 minutes, followed by a wash step with 5\% B from 25 to 30 minutes and then re-equilibration with $80 \%$ B from 25 to 30 minutes. Column oven was set to $20^{\circ} \mathrm{C}$, and a constant flow of solvents was set to $150 \mu \mathrm{L} / \mathrm{min}$. Samples were kept on $4^{\circ} \mathrm{C}$ autosampler throughout the measurements, and $5 \mu \mathrm{L}$ (retina) or $10 \mu \mathrm{L}$ (plasma) of sample was injected. Heated electrospray ionization (ESI) source settings used for the mass spectrometer were spray voltage 3500 (positive mode) and 2500 (negative mode), capillary temperature $250^{\circ} \mathrm{C}$, sheath gas 40 , and auxiliary gas 10 . Mass spectrometer method used was data-dependent MS2, where a full scan (survey) was recorded followed by MS2 scans for the top 5 peaks seen in the survey full scan. MS1 parameters were MS1 m/z window set from 70 to 1050 mass cutoff, resolution of 120,000, automatic gain control of $3 \times 10^{6}$ and maximum injection time of $100 \mathrm{~ms}$. MS2 values were recorded for the top 5 peaks after every MS1 scan, with 17,000 mass resolution, an automated gain control of $10^{5}$, maximum injection time of $50 \mathrm{~ms}$, and precursor ion isolation width of $2 \mathrm{~m} / \mathrm{z}$. A normalized collision energy of $40 \%$ was applied, a dynamic exclusion of 10 seconds was applied to exclude the redundant MS2 scans, isotope exclusion was applied, and underfill ratio of $1 \%$ was applied.

DMOG pharmacokinetics measurements were performed with a longer gradient method. The same machine, columns, and solvents were used, except that the LC method was as follows: $80 \%$ B to $20 \% \mathrm{~B}$ followed by washing with 5\% B from 31 to 39 minutes and final re-equilibration step with $80 \%$ B from 40 to 50 minutes. This LC method was run with a full-scan mass spectrometry method with positive-negative switching. The full scans were recorded with a mass range of 70 to $1050 \mathrm{~m} / z$ at a mass resolution of 60,000 , automatic gain control set to $3 \times 10^{6}$, and maximum injection time of $200 \mathrm{~ms}$. This method was adapted from Singh et al. (44). 
LC-MS data analysis. LC-MS data from ddMS2 measurement were converted to mzML format using compound discoverer (version 2.1, Thermo Fisher Scientific) and analyzed using XCMS Online tool (28). The metabolites were mapped onto the mouse database available on XCMS (28). Data were also analyzed with MSdial tool to confirm some of the metabolite annotations, using the library provided with the MSdial software $(45,46)$.

GC-MS measurement of metabolites. Lactate labeling from the media of retinal explants, and serine and glycine labeling from retinal intracellular extracts from explant cultures, were measured on GC (6890N, Agilent Technologies) coupled to EI mass selective detector (5973 MSD, Agilent Technologies) mass spectrometer using the method described in Singh et al. (15).

In vivo liver metabolite labeling samples were measured on GC (7890B, Agilent Technologies) coupled to EI/CI (extractor EI source) mass selective detector (5977B MSD, Agilent Technologies) mass spectrometer. $\mathrm{CI}$ ion source was set to a constant $70 \mathrm{eV}$ potential. Methane was used as a reagent gas, and CI was performed in positive mode ionization. CI source was held at a constant temperature of $300^{\circ} \mathrm{C}$ and quadrupole set at a constant temperature of $150^{\circ} \mathrm{C}$. Transfer line was held at $290^{\circ} \mathrm{C}$ and front-inlet temperature was $250^{\circ} \mathrm{C}$. GC column oven was set at $60^{\circ} \mathrm{C}$ starting temperature and was held for 1 minute. Temperature was ramped at a rate of $10^{\circ} \mathrm{C} / \mathrm{min}$ from $60^{\circ} \mathrm{C}$ to $325^{\circ} \mathrm{C}$ with a constant flow of helium gas at $1.1 \mathrm{~mL} / \mathrm{min}$. Agilent Technologies HP-5ms inert column $30 \mathrm{~m} \times 250 \mu \mathrm{m} \times 0.25 \mu \mathrm{m}$ was used for separation of derivatized metabolites.

All the data were analyzed using metabolite detector (47), MSD Chemstation (Agilent Technologies), and MassHunter Workstation Unknowns Analysis (Agilent Technologies) software. NIST17 (NIH) and Fiehn 2013 (Agilent Technologies) libraries were used for GC-MS data analysis.

Isocor software version 1.0 was used to derive mass isotopolog distributions for metabolites (48).

OIR model, RXD and MTX treatments, and retinal vasculature analysis. WT C57BL/6J mice were supplied by the Jackson Laboratory. OIR model was based on the previously described procedure developed by Smith (49) and was described in detail elsewhere (50-52). Briefly, newborn pups with their nursing dam were subjected to $75 \%$ oxygen in the Biospherix animal hyperoxia system (Biospherix Ltd.) from P7 to P12. RXD (10 $\mu \mathrm{g} / \mathrm{g}$ BW, Adooq) was injected i.p. at P6, P8, and P10, and MTX (0.2 $\mu \mathrm{g} / \mathrm{g}$ BW, Cayman Chemical) was given i.p. daily from P6 to P11. At P17 mouse retinas were isolated, fixed, and stained with isolectin GS-IB 4 Alexa 568 Conjugate (Life Technologies) as previously described (25). Oxygen-induced vascular pathology, i.e., vaso-obliteration and neovascularization, was automatically quantified using deep learning segmentation software available at a free, public, open-source repository (53). PCR to confirm tissue specificity of the HIF-1 KO was performed as described in Hoppe et al. (8).

Availability of data and material. The untargeted metabolomics data have been submitted to public repository Metabolights and study ID is MTBLS856.

Statistics. Statistical analysis for both GC and LC data were performed in R version 3.4.0 2017-04-21 (54) and GraphPad Prism 7 for most of the data, except for XCMS-analyzed data. A $P$ value of less than 0.05 was considered statistically significant. All error values are \pm SEM.

Study approval. All experimental procedures involving live animals were conducted in accordance with the guidelines of the NIH's Guide for the Care and Use of Laboratory Animals (National Academies Press, 2011) and approved by the Cleveland Clinic institutional animal care and use committee (IACUC, protocol 2016-1677). All animal experiments conformed to the rules and regulations of the Association of Research in Vision and Ophthalmology.

\section{Author contributions}

CS, GH, HB, and JES designed experiments. CS and GH performed experiments. LM and YB performed OIR model experiments. LM, YB, and WS performed retinal flat mounts. LM and YB were responsible for PCR. VT and AS performed sample processing for metabolomics analyses. CS, GH, HB, and JES interpreted the data. CS and JES wrote the paper. GH and HB edited the paper.

\section{Acknowledgments}

This study was supported by the National Eye Institute (R01 EY024972 and P30 EY025585); The Hartwell Foundation Biomedical Research Fellowship (HWF06092015); and Research to Prevent Blindness Physician Scientist (RPB 1801). We appreciate the use of the LCMS/MS, supported by the Cleveland Clinic metabolomics core, Belinda Willard and Maryam Goudarzi. 
Address correspondence to: Jonathan E. Sears, Cole Eye Institute, Cleveland Clinic, 9500 Euclid Ave., Cleveland, Ohio 44195, USA. Phone: 216.444.8157; Email: searsj@ccf.org.

1. Kim SJ, Port AD, Swan R, Campbell JP, Chan RVP, Chiang MF. Retinopathy of prematurity: a review of risk factors and their clinical significance. Surv Ophthalmol. 2018;63(5):618-637.

2. Park AM, Sanders TA, Maltepe E. Hypoxia-inducible factor (HIF) and HIF-stabilizing agents in neonatal care. Semin Fetal Neonatal Med. 2010;15(4):196-202.

3. Hartnett ME, Lane RH. Effects of oxygen on the development and severity of retinopathy of prematurity. JAAPOS 2013;17(3):229-234.

4. Ozaki H, et al. Hypoxia inducible factor-1 $\alpha$ is increased in ischemic retina: temporal and spatial correlation with VEGF expression. Invest Ophthalmol Vis Sci. 1999;40(1):182-189.

5. Semenza GL, Wang GL. A nuclear factor induced by hypoxia via de novo protein synthesis binds to the human erythropoietin gene enhancer at a site required for transcriptional activation. Mol Cell Biol. 1992;12(12):5447-5454.

6. Trichonas G, Lee TJ, Hoppe G, Au J, Sears JE. Prolyl hydroxylase inhibition during hyperoxia prevents oxygen-induced retinopathy in the rat 50/10 model. Invest Ophthalmol Vis Sci. 2013;54(7):4919-4926.

7. Sears JE, Hoppe G, Ebrahem Q, Anand-Apte B. Prolyl hydroxylase inhibition during hyperoxia prevents oxygen-induced retinopathy. Proc Natl Acad Sci U S A. 2008;105(50):19898-19903.

8. Hoppe G, et al. Comparative systems pharmacology of HIF stabilization in the prevention of retinopathy of prematurity. Proc Natl Acad Sci U S A. 2016;113(18):E2516-E2525.

9. Semenza GL. Regulation of physiological responses to continuous and intermittent hypoxia by hypoxia-inducible factor 1. Exp Physiol. 2006;91(5):803-806.

10. Kim JW, Tchernyshyov I, Semenza GL, Dang CV. HIF-1-mediated expression of pyruvate dehydrogenase kinase: a metabolic switch required for cellular adaptation to hypoxia. Cell Metab. 2006;3(3):177-185.

11. Semenza GL. Hypoxia-inducible factors: coupling glucose metabolism and redox regulation with induction of the breast cancer stem cell phenotype. EMBO J. 2017;36(3):252-259.

12. Fan J, Ye J, Kamphorst JJ, Shlomi T, Thompson CB, Rabinowitz JD. Quantitative flux analysis reveals folate-dependent NADPH production. Nature. 2014;510(7504):298-302.

13. Samanta D, Prabhakar NR, Semenza GL. Systems biology of oxygen homeostasis. Wiley Interdiscip Rev Syst Biol Med. 2017;9(4)

14. Samanta D, Park Y, Andrabi SA, Shelton LM, Gilkes DM, Semenza GL. PHGDH expression is required for mitochondrial redox homeostasis, breast cancer stem cell maintenance, and lung metastasis. Cancer Res. 2016;76(15):4430-4442.

15. Singh C, Sharma A, Hoppe G, Song W, Bolok Y, Sears JE. 3-Hydroxypyruvate destabilizes hypoxia inducible factor and induces angiostasis. Invest Ophthalmol Vis Sci. 2018;59(8):3440-3448.

16. Hoppe G, et al. Comparative systems pharmacology of HIF stabilization in the prevention of retinopathy of prematurity. Proc Natl Acad Sci U S A. 2016;113(18):E2516-E2525.

17. Kaelin WG, Ratcliffe PJ. Oxygen sensing by metazoans: the central role of the HIF hydroxylase pathway. Mol Cell. 2008;30(4):393-402.

18. Kaelin WG. Cancer and altered metabolism: potential importance of hypoxia-inducible factor and 2-oxoglutarate-dependent dioxygenases. Cold Spring Harb Symp Quant Biol. 2011;76:335-345.

19. Epstein AC, et al. C. elegans EGL-9 and mammalian homologs define a family of dioxygenases that regulate HIF by prolyl hydroxylation. Cell. 2001;107(1):43-54.

20. Wang GL, Semenza GL. General involvement of hypoxia-inducible factor 1 in transcriptional response to hypoxia. Proc Natl Acad Sci U S A. 1993;90(9):4304-4308.

21. Ivan M, et al. HIF $\alpha$ targeted for VHL-mediated destruction by proline hydroxylation: implications for $\mathrm{O} 2$ sensing. Science. 2001;292(5516):464-468.

22. Mole DR, et al. 2-oxoglutarate analogue inhibitors of HIF prolyl hydroxylase. Bioorg Med Chem Lett. 2003;13(16):2677-2680.

23. McDonough MA, et al. Cellular oxygen sensing: Crystal structure of hypoxia-inducible factor prolyl hydroxylase (PHD2). Proc Natl Acad Sci U S A. 2006;103(26):9814-9819.

24. Samanta D, Semenza GL. Maintenance of redox homeostasis by hypoxia-inducible factors. Redox Biol. 2017;13:331-335.

25. Hoppe G, et al. Inducing a visceral organ to protect a peripheral capillary bed: stabilizing hepatic HIF-1 $\alpha$ prevents oxygen-induced retinopathy. Am J Pathol. 2014;184(6):1890-1899.

26. Olenchock BA, et al. EGLN1 inhibition and rerouting of $\alpha$-ketoglutarate suffice for remote ischemic protection. Cell. 2016;165(2):497.

27. Provenzano R, et al. Roxadustat (FG-4592) versus epoetin alfa for anemia in patients receiving maintenance hemodialysis: a phase 2, randomized, 6- to 19-week, open-label, active-comparator, dose-ranging, safety and exploratory efficacy study. $\mathrm{Am} J$ Kidney Dis. 2016;67(6):912-924.

28. Forsberg EM, et al. Data processing, multi-omic pathway mapping, and metabolite activity analysis using XCMS Online. Nat Protoc. 2018;13(4):633-651.

29. Li S, et al. Predicting network activity from high throughput metabolomics. PLoS Comput Biol. 2013;9(7):e1003123.

30. National Library of Medicine. Hazardous Substances Data Bank Web site. https://toxnet.nlm.nih.gov/cgi-bin/sis/search/ a?dbs+hsdb:@term+@DOCNO+3123. Accessed June 25, 2019.

31. Fets L, et al. MCT2 mediates concentration-dependent inhibition of glutamine metabolism by MOG. Nat Chem Biol. 2018;14(11):1032-1042.

32. Hurley JB, Lindsay KJ, Du J. Glucose, lactate, and shuttling of metabolites in vertebrate retinas. J Neurosci Res. 2015;93(7):1079-1092.

33. Winkler BS. Glycolytic and oxidative metabolism in relation to retinal function. J Gen Physiol. 1981;77(6):667-692.

34. Vandekeere S, et al. Serine synthesis via PHGDH is essential for heme production in endothelial cells. Cell Metab. 2018;28(4):573-587.e13. 
35. Kalhan SC. One-carbon metabolism, fetal growth and long-term consequences. Nestle Nutr Inst Workshop Ser. 2013;74:127-138.

36. Scerri TS, et al. Genome-wide analyses identify common variants associated with macular telangiectasia type 2. Nat Genet. 2017;49(4):559-567

37. Khodabande A, et al. Management of idiopathic macular telangiectasia type 2. Ophthalmol Ther. 2019;8(2):155-175

38. Kory N, et al. SFXN1 is a mitochondrial serine transporter required for one-carbon metabolism. Science. 2018;362(6416):eaat9528.

39. Paris LP, et al. Global metabolomics reveals metabolic dysregulation in ischemic retinopathy. Metabolomics. 2016;12:15.

40. Pierce EA, Foley ED, Smith LE. Regulation of vascular endothelial growth factor by oxygen in a model of retinopathy of prematurity. Arch Ophthalmol. 1996;114(10):1219-1228.

41. Alon T, Hemo I, Itin A, Pe'er J, Stone J, Keshet E. Vascular endothelial growth factor acts as a survival factor for newly formed retinal vessels and has implications for retinopathy of prematurity. Nat Med. 1995;1(10):1024-1028

42. Ashton N, Ward B, Serpell G. Effect of oxygen on developing retinal vessels with particular reference to the problem of retrolen tal fibroplasia. Br J Ophthalmol. 1954;38(7):397-432.

43. Du J, Linton JD, Hurley JB. Probing metabolism in the intact retina using stable isotope tracers. Meth Enzymol. 2015;561:149-170.

44. Singh C, Glaab E, Linster CL. Molecular identification of d-ribulokinase in budding yeast and mammals. $J$ Biol Chem. 2017;292(3):1005-1028.

45. Tsugawa H, et al. MS-DIAL: data-independent MS/MS deconvolution for comprehensive metabolome analysis. Nat Methods. 2015;12(6):523-526

46. Lai Z, et al. Identifying metabolites by integrating metabolome databases with mass spectrometry cheminformatics. Nat Methods. 2018;15(1):53-56.

47. Hiller K, Hangebrauk J, Jäger C, Spura J, Schreiber K, Schomburg D. MetaboliteDetector: comprehensive analysis tool for targeted and nontargeted GC/MS based metabolome analysis. Anal Chem. 2009;81(9):3429-3439.

48. Millard P, Letisse F, Sokol S, Portais JC. IsoCor: correcting MS data in isotope labeling experiments. Bioinformatics. 2012;28(9):1294-1296.

49. Smith LE, et al. Oxygen-induced retinopathy in the mouse. Invest Ophthalmol Vis Sci. 1994;35(1):101-111.

50. Sears JE, Hoppe G, Ebrahem Q, Anand-Apte B. Prolyl hydroxylase inhibition during hyperoxia prevents oxygen-induced retinopathy. Proc Natl Acad Sci U S A. 2008;105(50):19898-19903.

51. Hoppe G, et al. Inducing a visceral organ to protect a peripheral capillary bed: stabilizing hepatic HIF-1 $\alpha$ prevents oxygen-induced retinopathy. Am J Pathol. 2014;184(6):1890-1899.

52. Singh C, Sharma A, Hoppe G, Song W, Bolok Y, Sears JE. 3-Hydroxypyruvate destabilizes hypoxia inducible factor and induces angiostasis. Invest Ophthalmol Vis Sci. 2018;59(8):3440-3448.

53. Xiao S, et al. Fully automated, deep learning segmentation of oxygen-induced retinopathy images. JCI Insight. 2017;2(24):97585

54. R: A Language and Environment for Statistical Computing [computer program]. Vienna, Austria: R Foundation for Statistical Computing; 2017. 